\title{
Clinical characteristics and HLA genotypes in Chinese patients with anti-SLA/LP-positive autoimmune hepatitis
}

\author{
Hai-Ping Zhang ${ }^{1}$, Yan-Min Liu ${ }^{2}$, Zhao Li ${ }^{3}$, Yin-Xue Ma ${ }^{1}$, Li-Juan Li ${ }^{1}$, Dan-Tong Zhao ${ }^{1}$, Jin-Li Lou ${ }^{1}$, \\ Zu-Hua Gao ${ }^{4}$, Hui-Ping Yan ${ }^{1}$ \\ ${ }^{1}$ Clinical Laboratory Center and Clinical Research Center for Autoimmune Liver Disease, Beijing Youan Hospital, Capital Medical University, \\ Beijing, China; ${ }^{2}$ Department of Liver Disease Immunology, Beijing Youan Hospital, Capital Medical University, Beijing, China; ${ }^{3}$ CAS Key \\ Laboratory of Mental Health, Institute of Psychology, Chinese Academy of Sciences, Beijing, China; ${ }^{4}$ Department of Pathology, McGill University, \\ Montreal, Canada \\ Contributions: (I) Conception and design: HP Yan, ZH Gao, HP Zhang; (II) Administrative support: HP Yan, ZH Gao, JL Lou; (III) Provision of \\ study materials or patients: HP Zhang, YM Liu; (IV) Collection and assembly of data: HP Zhang, YX Ma, LJ Li, DT Zhao; (V) Data analysis and \\ interpretation: HP Zhang, Z Li; (VI) Manuscript writing: All authors; (VII) Final approval of manuscript: All authors. \\ Correspondence to: Hui-Ping Yan. Clinical Laboratory Center and Clinical Research Center for Autoimmune Liver Disease, Beijing Youan Hospital, \\ Capital Medical University, Beijing 100069, China. Email: bjyhp503@ccmu.edu.cn; Zu-Hua Gao. Department of Pathology, McGill University, \\ Montreal, QC, Canada. Email: zu-hua.gao@mcgill.ca.
}

Background: Anti-soluble liver antigen/liver pancreas (anti-SLA/LP) is a highly specific serological marker for the diagnosis of autoimmune hepatitis (AIH). The aim of the present study was to define the clinical characteristics and human leucocyte antigen (HLA) genotypes of Chinese patients with anti-SLA/LP positive AIH.

Methods: Ninety-one AIH patients who were anti-SLA/LP positive were enrolled in this case control study. Clinical information was obtained through reviewing patients' clinical notes. High-resolution genotyping of HLA-A, B, C, DRB1, and DQB1 alleles was performed by sequence-based typing polymerase chain reaction on 62 of the 91 patients. Data from 500 healthy patients were used as baseline controls.

Results: Anti-SLA/LP-positive AIH patients were characterized as follows: adults (age 20-80 years), female (88\%), and frequent anti-nuclear antibody positivity (91\%). Genetically, compared with the controls, HLA-B*35:01 and $C^{*} 08: 01$ were significantly more frequent in patients. The frequencies of HLA-B*08:01, B*40:02, DRB1*04:01, DRB1*04:05, DRB1*14:01, and DRB1*16:02 increased, and the frequency in DRB1*15:01 decreased in patients, but did not reach significance after Bonferroni's correction. Patients with other autoimmune diseases had a higher DRB1*04:05 and DQB1*04:01 allele carrier frequency than those without. DRB1*04:05 and DQB1*04:01 alleles were found at increased frequency in patients with decompensated liver disease than those with compensated liver disease.

Conclusions: Chinese anti-SLA/LP-positive AIH patients have some distinct clinical characteristics than other populations reported in the literature. The presence of certain specific HLA alleles could potentially increase the risk of developing anti-SLA/LP-positive AIH or other autoimmune disease and decompensated liver disease in the Chinese population.

Keywords: Autoimmune hepatitis (AIH); anti-soluble liver antigen/liver pancreas; human leucocyte antigen (HLA)

Submitted Nov 21, 2020. Accepted for publication Jan 08, 2021.

doi: $10.21037 /$ atm-20-8036

View this article at: http://dx.doi.org/10.21037/atm-20-8036 


\section{Introduction}

Autoimmune hepatitis (AIH) is an autoimmune disease characterized by liver parenchymal cell damage. Patients are mostly female, with elevated liver enzymes, immunoglobulin $\mathrm{G}(\mathrm{IgG})$, multiple autoantibodies in the serum, and lymphoplasmacytic inflammation in the liver tissues (1-3). The International Autoimmune Hepatitis Group (IAIHG) has established comprehensive criteria for the diagnosis of AIH, which combines serological, clinical, and pathological parameters after excluding other liver diseases, such as viral hepatitis, alcoholic liver disease, and drug-induced liver damage (4). The presence of autoantibodies in patients' serum is an important criterion for the diagnosis of AIH. Anti-nuclear antibody (ANA), anti-smooth muscle antibody (ASMA), anti-liver kidney microsomal antibody type 1 (anti-LKM1), and/or anti-liver cytosol type 1 (anti-LC1) autoantibodies constitute the conventional serological repertoire for the diagnosis of AIH $(5,6)$. ANA and ASMA are detected in $80 \%$ and $63 \%$ of white North American adults with AIH at presentation, respectively. ANA and ASMA can also occur as an isolated serological finding in other liver diseases, such as primary sclerosing cholangitis, chronic hepatitis C, and hepatitis B. Serum anti-SLA/LP, as non-conventional autoantibodies, has been shown to be a highly specific biomarker for the diagnosis of AIH, but its prevalence in AIH patients is only $10-20 \%(1,7-10)$. A multicenter retrospective study of autoimmune liver disease in China showed that only 6\% (16/248) of AIH patients were anti-SLA/LP positive (3). However, only a few reports have been published, and these have only involved a small number of cases; therefore, the clinical characteristics of anti-SLA/LP-positive Chinese AIH patients have not been well documented $(9,11,12)$.

The general consensus in the scientific community is that AIH results from the combination of genetic and environmental factors. Genetic susceptibility to AIH is strongly associated with human leucocyte antigen (HLA) genes. Czaja et al. reported that the HLA-DRB1*03:01 allele in white North American patients was associated with disease occurrence and severity by affecting autoantigen presentation and/or by synergizing with other autoimmune promoters (13). Few studies have addressed the frequency and significance of specific HLA in selected groups of antiSLA/LP-positive AIH patients. It has been shown that HLA DR3 functions as an independent genetic risk factor for the development of anti-SLA/LP-positive type $1 \mathrm{AIH}$ in Europe (13-15). However, the role of HLA polymorphisms in Chinese AIH patients with anti-SLA/LP has never been sufficiently evaluated.

In the present study, we first analyzed the clinical characteristics of 91 Chinese AIH patients with anti-SLA/ LP. We then evaluated the associations between HLA class I (A, B, C) and II (DRB1, DQB1) gene polymorphisms and disease susceptibility in the largest cohort of Chinese antiSLA/LP-positive AIH patients to date. Our data provide new insight into the correlation of specific HLA alleles with the risk of anti-SLA/LP-positive AIH in the Chinese population.

We present the following article in accordance with the STROBE reporting checklist (available at http://dx.doi. org/10.21037/atm-20-8036).

\section{Methods}

\section{Patients}

Between January 2003 and August 2019, 110 patients with anti-SLA/LP-positive AIH were recruited from Beijing Youan Hospital. Of these patients, 12 had a diagnosis of overlapping $\mathrm{AIH}$ and primary biliary cholangitis (PBC) and 7 patients had positive hepatitis B virus (HBV) and/or hepatitis $\mathrm{C}$ virus (HCV) virology; these 19 patients were excluded from the present study (Figure 1). The remaining 91 patients with anti-SLA/LP-positive AIH were enrolled in this case control study. All procedures performed in this study involving human participants were in accordance with the Declaration of Helsinki (as revised in 2013). The Institutional Ethics Review Board of Beijing Youan Hospital, Capital Medical University, approved the study (No. [2012]44). Informed consent was obtained from each participant. AIH diagnosis was made based on the diagnosed criteria for $\mathrm{AIH}(3,4,7,16)$. Liver biopsy was available in 39 anti-SLA/LP-positive patients.

\section{Serological assays}

Serum anti-SLA/LP was determined by commercially available enzyme-linked immunosorbent assay (ELISA kits (EUROIMMUN, Lubeck, Germany) following the manufacturer's instructions. ELISA results were classified as positive when values $>20 \mathrm{RU} / \mathrm{mL}$. Other autoantibodies, including ANA, ASMA, and anti-LKM were measured by indirect immunofluorescence (EUROIMMUN, Germany). A titer $\geq 1: 100$ was interpreted as positive. Anti-LC1 was measured by western blot (Shenzhen YHLO Biotech, 


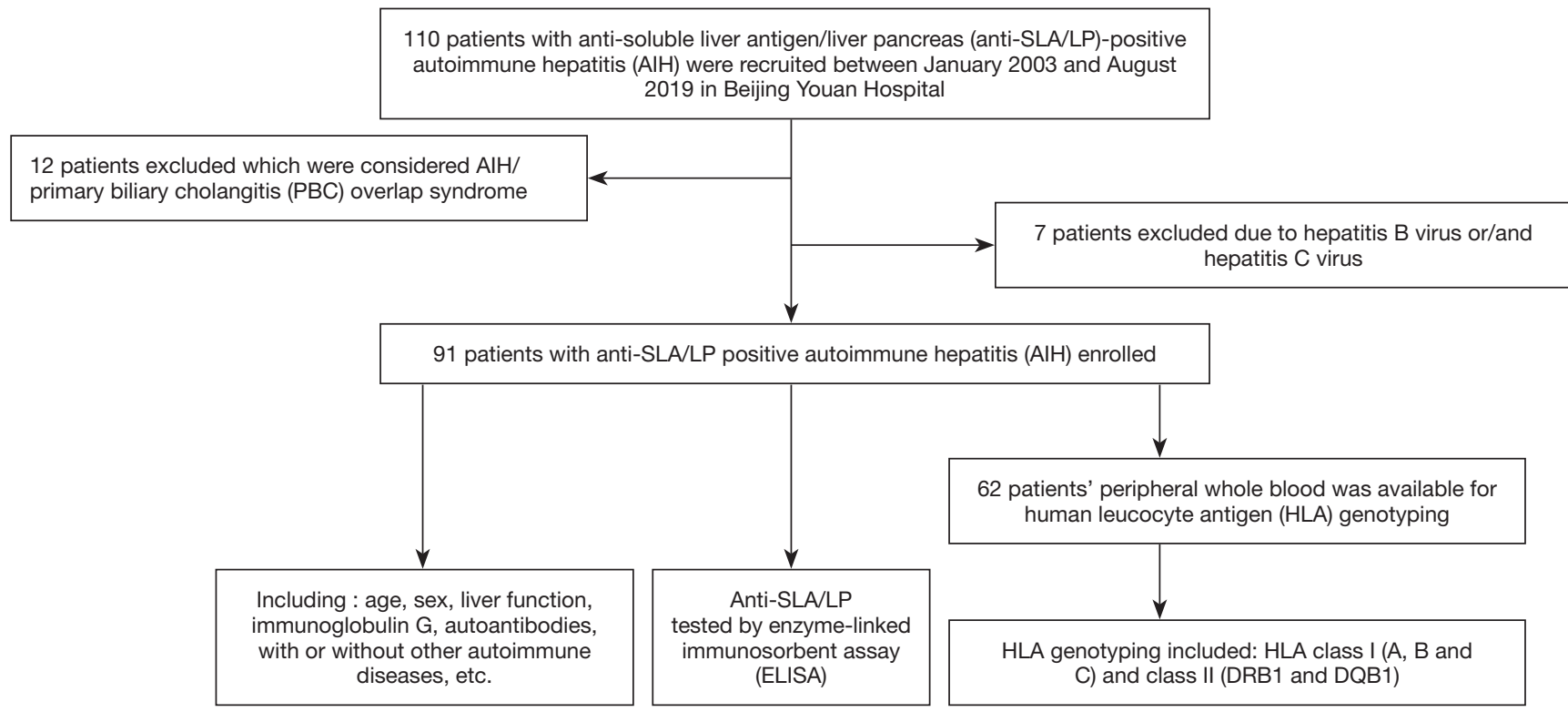

Figure 1 Flowchart of the search and identification of patients with anti-soluble liver antigen/liver pancreas (anti-SLA/LP)-positive autoimmune hepatitis (AIH).

Shenzhen, China).

All samples were tested for hepatitis B surface antigen and anti-HCV (HCV) antibodies by chemiluminescence using the Cobase 601 Analyzer (Roche Diagnostics GmbH, Mannheim, Germany) according to the manufacturer's protocols.

\section{HLA class genotyping}

In 62 of the 91 anti-SLA/LP-positive AIH patients, peripheral whole blood was available for HLA genotyping. Five hundred healthy patients who were used in a previous study were adopted as controls (17). Genomic DNA was purified from peripheral whole blood using the hydrochloride method, as described previously $(17,18)$. The HLA class I (A, B, and C) and class II (DRB1 and DQB1) allelic genotypes were determined using sequence-based typing polymerase chain reaction at the Beijing Genomics Institute, Shenzhen (BGI Shenzhen, Shenzhen, China) (17).

\section{Statistical analysis}

SPSS version 17.0 software (SPSS, Chicago, IL, USA) was used for the statistical analysis. Results were expressed as median (range). Allele distributions were compared between patients and controls using the $\chi^{2}$-test or Fisher's exact tests. Two-sided $\mathrm{P}<0.05$ was considered statistically significant. For multiple testing, $\mathrm{P}$ values were corrected $(\mathrm{Pc})$ by the number of comparisons, according to Bonferroni's inequality method. Association strengths were estimated by calculating the odds ratio and $95 \%$ confidence interval.

\section{Results}

\section{Clinical characteristics of the 91 AIH patients with anti- $S L A / L P$}

The clinical information of the 91 anti-SLA/LP-positive AIH patients is shown in Table 1. The patient population was characterized as follows: adults (age 20-80 years), female $(88 \%)$, with frequent concurrent positive serum ANA and negative anti-LKM and anti-LC1. More than one-third of patients had decompensated liver cirrhosis and acute-on-chronic hepatic failure.

Twenty-two patients had other concurrent autoimmune diseases, including hypothyroidism $(\mathrm{n}=8,8.8 \%)$, Sjogren syndrome $(n=4,4.4 \%)$, systemic lupus erythematous $(n=3$, $3.3 \%)$, interstitial pneumonitis $(n=3,3.3 \%)$, rheumatoid arthritis $(\mathrm{n}=2,2.2 \%)$, autoimmune anemia $(\mathrm{n}=1,1.1 \%)$, and type 1 diabetes mellitus $(\mathrm{n}=1,1.1 \%)$. As shown in Table 2, the level of albumin in those with other autoimmune diseases was lower than those with AIH alone. There were no significant differences in age, sex, alanine aminotransferase (ALT), aspartate aminotransferase 
Table 1 Clinical characteristic of anti-soluble liver antigen-/liver pancreas (anti-SLA/LP)-positive autoimmune hepatitis patients

\begin{tabular}{|c|c|}
\hline Clinical parameters & Value \\
\hline Age (year), median (range) & 55 [20-80] \\
\hline \multicolumn{2}{|l|}{ Sex, n (\%) } \\
\hline Female & $80(87.9)$ \\
\hline Male & $11(12.1)$ \\
\hline ALT (U/L), median (range) & $77.6(42.0-2,181)$ \\
\hline AST (U/L), median (range) & $99.8(43.7-1,883)$ \\
\hline TBil ( $\mu \mathrm{mol} / \mathrm{L})$, median (range) & $27.5(0.56-508.1)$ \\
\hline ALB (g/L), median (range) & $35.4(11.3-47.9)$ \\
\hline GLOB (g/L), median (range) & $37.9(21.3-170.8)$ \\
\hline$\gamma$-GT (U/L), median (range) & $65.2(3.67-1,044.7)$ \\
\hline ALP (U/L), median (range) & $106.8(43.8-1,906.3)$ \\
\hline $\lg G(g / d l)$, median (range) & $23(9.44-71.4)$ \\
\hline ANA $\geq 1: 100, n(\%)$ & $83(91.2)$ \\
\hline ASMA $\geq 1: 100, n(\%)$ & $15(16.5)$ \\
\hline AMA $\geq 1: 100, n(\%)$ & $8(8.8)$ \\
\hline Anti-SLA/LP $\geq 20$ RU/mL, n (\%) & $91(100.0)$ \\
\hline Anti-LKM $\geq 1: 100, n(\%)$ & $0(0.0)$ \\
\hline Anti-LC1 positive, $\mathrm{n}(\%)$ & $0(0.0)$ \\
\hline End-stage liver disease cases, $n(\%)$ & $31(34.1)$ \\
\hline Decompensated stage of cirrhosis & $24(26.4)$ \\
\hline Progression to hepatic failure & $5(5.5)$ \\
\hline Liver transplantation & $1(1.1)$ \\
\hline Death from liver failure & $1(1.1)$ \\
\hline
\end{tabular}

Values are expressed as median (range) unless otherwise noted. ALB, albumin; ALP, alkaline phosphatase; ALT, alanine aminotransferase; AMA, anti-mitochondrial antibody; ANA, anti-nuclear antibody; anti-LC1, anti-liver cytosol type 1 antibody; anti-LKM, anti-liver and kidney microsomal antibody; ASMA, anti-smooth muscle antibody; AST, aspartate aminotransferase; GLOB, globulin; $\gamma$-GT, gamma-glutamyl transpeptidase; IgG, immunoglobulin G; TBil, total bilirubin.

(AST), total bilirubin (TBil), globulin (GLOB), gammaglutamyl transpeptidase $(\gamma-\mathrm{GT})$, alkaline phosphatase (ALP), and immunoglobulin G ( $\mathrm{IgG}$ ) between patients with superimposed other autoimmune diseases and those without.

A positive anti-SLA/LP ELISA based on the manufacturer's cutoff of 20 RU was identified in 91 patients' sera. Sixty-five patients (65/91, 71.4\%) had a strong positive anti-SLA/LP value of $\geq 200 \mathrm{RU} / \mathrm{mL}$. Patients were divided into 3 different age groups: group 1: 20-40 years, group 2: 41-60 years, and group 3: 61-80 years. The distribution of anti-SLA/LP in the patient population among different age groups is shown in Figure 2. Most anti-SLA/LP-positive AIH patients $(55 / 91,60.4 \%)$ were in the $41-60$-year-old group. The median anti-SLA/LP titer showed a downward trend with increasing age.

\section{HLA genotype characteristics of anti-SLA/LP-positive AIH patients}

In 62 of the 91 anti-SLA/LP-positive AIH patients, peripheral whole blood was available for high-resolution genotyping of HLA class I (A, B, and C) and II (DRB1 and DQB1). The genotype frequencies were then compared with those of 500 previously genotyped healthy patients (15).

As seen in Table 3, among the HLA class I alleles, the frequency of HLA-B*35:01 and $C^{*}$ 08:01 was significantly higher in anti-SLA/LP-positive AIH patients than healthy patients $(22.6 \%$ vs. $8.2 \%, \mathrm{P}=0.0011 / \mathrm{Pc}=0.0332 ; 32.3 \%$ vs. $12.8 \%, \mathrm{P}=0.0002 / \mathrm{Pc}=0.0041$, respectively). There was a trend of higher representation of HLA-B*08:01 and $B * 40: 02$ alleles in anti-SLA/LP-positive AIH patients than healthy patients $(8.1 \%$ vs. $1.6 \%, \mathrm{P}=0.0089 / \mathrm{Pc}=0.1294$; $11.3 \%$ vs. $4 \%, \mathrm{P}=0.0211 / \mathrm{Pc}=0.12942039$, respectively). Among the HLA class II alleles, anti-SLA/LP-positive AIH patients showed a clear trend of higher frequency in DRB1*04:01, DRB1*04:05, DRB1*14:01, and DRB1*16:02 alleles compared with healthy patients $(8.1 \%$ vs. $1.6 \%$, $\mathrm{P}=0.0089 / \mathrm{P} \mathrm{c}=0.2139 ; 21.0 \%$ vs. $10.4 \%, \mathrm{P}=0.0201 /$ $\mathrm{P} c=0.2139 ; 3.2 \%$ vs. $0.2 \%, \mathrm{P}=0.0334 / \mathrm{Pc}=0.2139 ; 12.9 \%$ vs. $5.2 \%, \mathrm{P}=0.0411 / \mathrm{Pc}=0.2139$, respectively), and a clear trend of lower frequency in the DRB1*15:01 HLA allele (14.5\% vs. $27.4 \%, \mathrm{P}=0.0312 / \mathrm{Pc}=0.2139$ ) (Table 4). There was no significant difference in the HLA-DQB1 allele frequency between anti-SLA/LP-positive AIH patients and healthy patients.

We further analyzed the associations between HLA and the patients' clinical and laboratory parameters, including age, sex, titer of anti-SLA/LP, compensated versus decompensated liver disease status, and if the patients had other concomitant autoimmune diseases or not. There was no significant difference in the frequencies of HLA alleles among groups of different age and sex (data not shown). Among the groups of different titer of anti-SLA/LP, the HLA-A $* 33: 03$ allele frequency increased in the titer of 
Table 2 Comparison of clinical and laboratory findings between those with and without other autoimmune diseases in an anti-soluble liver antigen/liver pancreas (anti-SLA/LP)-positive autoimmune hepatitis patient population

\begin{tabular}{|c|c|c|c|}
\hline Features & With autoimmune diseases $(n=22)$ & Without autoimmune diseases $(n=69)$ & $P$ value \\
\hline Sex (female/male) & $18 / 4$ & $65 / 4$ & 0.176 \\
\hline Anti-SLA/LP (RU/mL), median (range) & $291.0(21.5-543.3)$ & $295.9(20.3-544.1)$ & 0.184 \\
\hline ALT (U/L), median (range) & $43.0(49.4-2,005.2)$ & $90.8(42.0-2,181.0)$ & 0.205 \\
\hline TBil ( $\mu \mathrm{mol} / \mathrm{L})$, median (range) & $33.4(0.53-482.2)$ & $24.5(0.56-508.1)$ & 0.337 \\
\hline ALB (g/L), median (range) & $32.1(15.9-44.4)$ & $37.3(11.3-47.9)$ & $0.013^{*}$ \\
\hline GLOB (g/L), median (range) & $39.8(29.0-68.1)$ & $37.8(21.3-96.7)$ & 0.422 \\
\hline$\gamma$-GT (U/L), median (range) & $55.0(3.82-397.2)$ & $83.9(3.67-1,044.7)$ & 0.241 \\
\hline
\end{tabular}

${ }^{*}, \mathrm{P}<0.05$. Values are expressed as median (range) unless otherwise noted. ALB, albumin; ALP, alkaline phosphatase; ALT, alanine aminotransferase; AST, aspartate aminotransferase; GLOB, globulin; $\gamma$-GT, gamma-glutamyl transpeptidase; lgG, immunoglobulin G; TBil, total bilirubin.

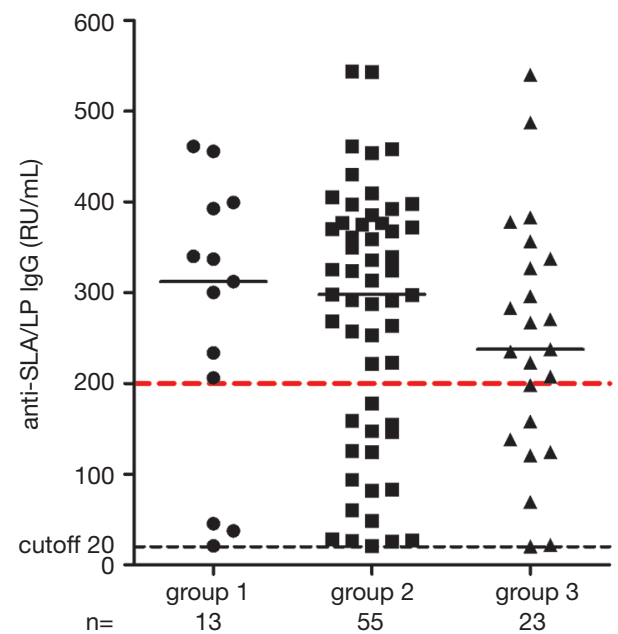

Figure 2 Scatterplot of anti-soluble liver antigen/liver pancreas immunoglobulin $\mathrm{G}$ enzyme-linked immunosorbent assay results for different age groups. Group 1: 20-40 years, group 2: 41-60 years, group 3: 61-80 years. Black dotted line indicates cutoff of 20 units.

$20-200 \mathrm{RU} / \mathrm{mL}$ than that of $\geq 200 \mathrm{RU} / \mathrm{mL}$ (25.9\% vs. $2.9 \%$, $\mathrm{P}=0.0168)$, the $\mathrm{DQB} 1{ }^{*} 03: 02$ allele frequency decreased in the titer of $20-200 \mathrm{RU} / \mathrm{mL}$ than that of the $\geq 200 \mathrm{RU} / \mathrm{mL}$ group (0 vs. $22.9 \%, \mathrm{P}=0.0076$ ) (Table S1). The HLA-B*15:18, DRB $1 * 04: 05$, and DQB $1 * 04: 01$ alleles were found at an increased frequency in patients with decompensated liver diseases than those with compensated liver diseases ( $17.6 \%$ vs. $0, \mathrm{P}=0.0180 ; 52.9 \%$ vs. $8.9 \%, \mathrm{P}=0.0005$; $35.3 \%$ vs. $8.9 \%, \mathrm{P}=0.0200$, respectively) (Table S2). Anti-SLA/LP-positive AIH patients with other concomitant autoimmune diseases showed a trend of higher HLA-B*13:01, DRB1*04:05, and DQB1*04:01 allele carrier frequency than those without other autoimmune diseases (18.8 vs. $2.2 \%, \mathrm{P}=0.0490 ; 43.8 \%$ vs. $13 \%, \mathrm{P}=0.0280 ; 37.5 \%$ vs. $8.7 \%, \mathrm{P}=0.0140$, respectively) (Table $\mathrm{S} 3$ ).

\section{Discussion}

In 2000, Wies et al. provided evidence that anti-SLA and anti-LP were one and the same autoantibody (antiSLA/LP) against a synthase converting O-phosphoseryltRNA to selenocysteinyl-tRNA (19). Structurally, SLA/ LP has sequence homology with short segment of human asialoglycoprotein receptor (ASGPR), and this segment forms part of a hydrophobic membrane-spanning region. When SLA/LP is present on the cell membrane of hepatocytes, it becomes a target of immune cells (13). Determining the clinical characteristics of the associated susceptible HLA alleles could help us gain a better understanding of the pathogenesis of the unique population of anti-SLA/LP-positive AIH patients.

According to the American Association for the Study 
Table 3 Statistical comparison of representative human leucocyte antigen (HLA)-A,B,C allele frequency between anti-soluble liver antigen-/liver pancreas (anti-SLA/LP)-positive autoimmune hepatitis (AIH) patients and controls

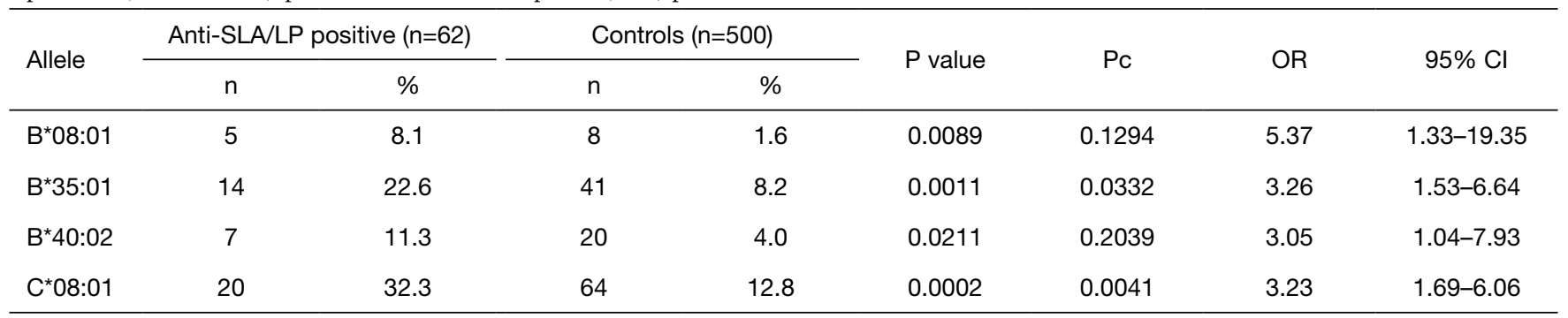

Associations were tested by Fisher's extract test using $2 \times 2$ contingency tables. OR and its $95 \% \mathrm{Cl}$ for the carrier of HLA were calculated in two groups. Pc values were calculated using Bonferroni's procedure. HLA-A-C alleles detected at similar frequencies in AlH patients with anti-SLA/LP and normal controls are not shown. $\mathrm{Cl}$, confidence interval; OR, odds ratio; Pc, corrected $\mathrm{P}$ value.

of Liver Diseases (6,20), AIH is generally divided into two types. AIH type 1 accounts for $95-96 \%$ of patients, mainly adults, with positive ANA and/or ASMA serology. Anti-SLA/LP-positive patients are included in this type. AIH type 2 accounts for $4-5 \%$ of patients, mainly children and adolescents, with positive anti-LKM1 and antiLC1 serology. Mieli-Vergani et al. reported that a small population of pediatric patients also have serum antiSLA/LP, and suggested that anti-SLA/LP can be in both types of AIH (1). In the current study, 110 anti-SLA/LPpositive patients from 850 diagnosed AIH patients (from the medical record database) were recruited between 2003 and 2019; anti-SLA/LP was present in 12.9\% (110/850) of AIH. Among 110 patients with anti-SLA/LP-positive AIH, 12 patients with the diagnosis of overlapping $\mathrm{AIH}$ and $\mathrm{PBC}$ and 7 patients with positive $\mathrm{HBV}$ and/or HCV virology were excluded. The remaining 91 patients with anti-SLA/ LP-positive AIH were enrolled in this case control study. All patients were $>18$ years old. ANA $(91.2 \%)$ or ASMA $(16.5 \%)$ was detectable in the 91 patients with anti-SLA/ LP, while anti-LKM or anti-LC1 was not detected. That is, anti-SLA/LP-positive patients were only seen in $\mathrm{AIH}$ type 1 , and not in AIH type 2. This phenomenon may be related to the low proportion of pediatric patients in our study population. As shown in Figure 2, the age distribution of anti-SLA/LP-positive cases in the present study shows a peak of 41-60 years, which is different to the AIH cases (including both types of AIH) reported in Denmark in a previously published study (21). In addition, more than one-third of the patients $(34.1 \%)$ had decompensated liver cirrhosis and acute-on-chronic hepatic failure, and about one-quarter of the patients (24.2\%) had other autoimmune diseases, which is characteristic of AIH patients with antiSLA/LP.
The pathogenesis of AIH is still unclear. Like many other autoimmune diseases, genetic susceptibility is considered to be one of the important pathogenic factors of AIH. HLA is the most important genetic risk factor for $\mathrm{AIH}$, and may influence disease occurrence and severity by affecting autoantigen presentation. Strettell et al. reported the association between HLA-C*07:01 allele and type 1 AIH in white patients (22). Umemura et al. demonstrated the association between HLA-C ${ }^{*}$ 01:02 allele with type 1 AIH in a Japanese population (23). In the present study, we found that the frequency of HLA-B*35:01 and $C^{*} 08: 01$ alleles were significantly higher in anti-SLA/LP-positive AIH patients than normal patients. HLA-C alleles have been known to play a critical role in the autoimmune disease process through interaction with natural killer cells or cytotoxic T lymphocytes (22). Our findings on the association of HLA $C^{*}$ 08:01 alleles with anti-SLA/LPpositive AIH support this.

The association between HLA-DRB1 and the risk of developing type I AIH has been reported. In European and US populations, HLA-DRB1*03:01 and DRB1*04:01 are the most significant disease susceptible alleles of AIH-1 (24-26). These risk alleles were then confirmed in a of northern European large genome-wide association study (27). The presence of DRB1*04:05 was shown to increase the risk of AIH type 1 in Japanese and Chinese patients $(23,28)$. In Spanish AIH patients, the HLA-DRB1*14:01 allele significantly increased the risk of AIH-1 (29). The DRB1*15:01 allele reduced the risk of AIH-1 in Japanese and European populations $(20,23)$. Few studies have reported the association between HLA genotypes and serum autoantibodies. In AIH-2 patients, the frequency of the HLA-DRB $1 * 03$ allele is significantly higher in patients with both anti-LKM1 and anti-LC1 or in patients with only anti- 
Table 4 Human leucocyte antigen (HLA)-DRB1 allele carrier frequency in patients with anti-soluble liver antigen-/liver pancreas (anti-SLA/ $\mathrm{LP})$-positive autoimmune hepatitis (AIH) patients and controls

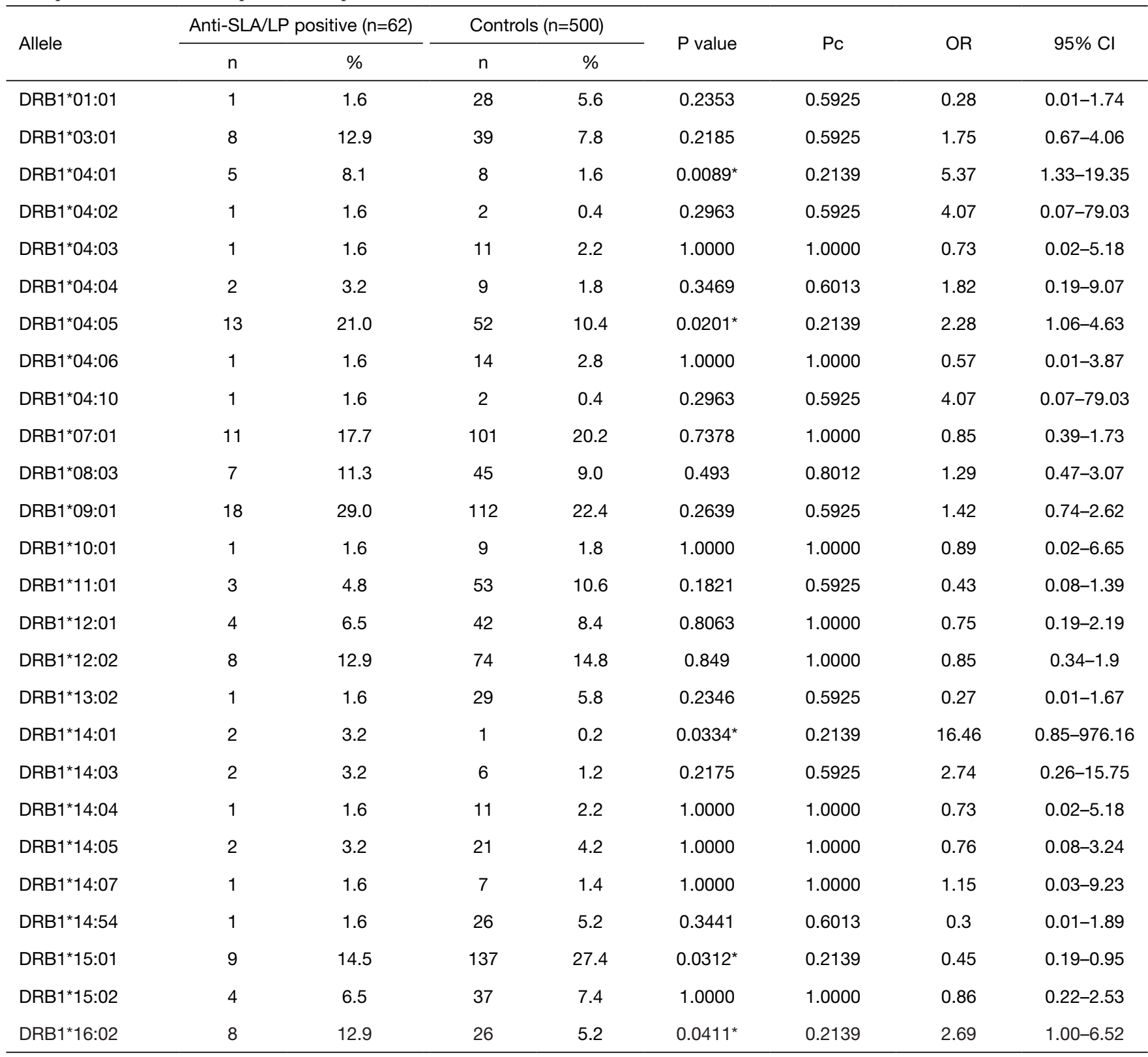

*, $\mathrm{P}<0.05$. Associations were tested by Fisher's extract test using $2 \times 2$ contingency tables. OR and its $95 \% \mathrm{Cl}$ for the carrier of $\mathrm{HLA}$ were calculated in two groups. Pc values were calculated using Bonferroni's procedure. $\mathrm{Cl}$, confidence interval; OR, odds ratio; Pc, corrected $\mathrm{P}$ value.

LC1 compared with patients with only anti-LKM1. The frequency of the HLA-DRB $1{ }^{*} 07$ allele was higher in AIH2 patients who have only anti-LKM1 $(30,31)$. In the present study, we found that the frequencies of HLA-B*08:01, $\mathrm{B}^{*} 40: 02, \mathrm{DRB} 1{ }^{*} 04: 01, \mathrm{DRB} 1 * 04: 05, \mathrm{DRB} 1 * 14: 01$, and DRB1*16:02 increased, and DRB1*15:01 decreased in
Chinese AIH patients with anti-SLA/LP. The frequency of HLA-DRB $1 * 04: 05$ and DQB1*04:01 alleles increased in patients with decompensated liver diseases than those with compensated liver diseases, the frequency of DRB1*04:05 and DQB $1 * 04: 01$ alleles was higher in anti-SLA/LPpositive AIH patients with other autoimmune diseases than 
those with AIH alone. However, these associations did not reach statistical significance after Bonferroni's correction. This might be attributed to the small sample size and should not be overlooked due to its important mechanistic and clinical implications.

In summary, we described the clinical characteristics and frequency of HLA class I and class II alleles in the largest series to date of anti-SLA/LP-positive Chinese AIH patients. In our study population, there was a statistically significant clear association between the presence of HLA-B*35:01 and $C^{*} 08: 01$ and the risk of developing of anti-SLA/LP-positive AIH. There was also a trend of higher frequency of certain HLA DR alleles in Chinese anti-SLA/LP-positive AIH patients with decompensated liver diseases and with other concurrent autoimmune diseases. If validated in multicenter large-scale studies, these disease-specific HLA alleles could potentially become diagnostic genetic markers and targets of gene therapy.

\section{Acknowledgments}

Funding: This work was supported by the Development Center for Medical Science and Technology, National Health, and Family Planning Commission of the People's Republic of China (Grant No. 28-5-5).

\section{Footnote}

Reporting Checklist: The authors have completed the STROBE reporting checklist. Available at http://dx.doi. org/10.21037/atm-20-8036

Data Sharing Statement: Available at http://dx.doi. org/10.21037/atm-20-8036

Conflicts of Interest: All authors have completed the ICMJE uniform disclosure form (available at http://dx.doi. org/10.21037/atm-20-8036). The authors have no conflicts of interest to declare.

Ethical Statement: The authors are accountable for all aspects of the work in ensuring that questions related to the accuracy or integrity of any part of the work are appropriately investigated and resolved. All procedures performed in this study involving human participants were in accordance with the Declaration of Helsinki (as revised in 2013). The Institutional Ethics Review Board of Beijing Youan Hospital, Capital Medical University, approved the study (No. [2012]44). Informed consent was obtained from each participant.

Open Access Statement: This is an Open Access article distributed in accordance with the Creative Commons Attribution-NonCommercial-NoDerivs 4.0 International License (CC BY-NC-ND 4.0), which permits the noncommercial replication and distribution of the article with the strict proviso that no changes or edits are made and the original work is properly cited (including links to both the formal publication through the relevant DOI and the license). See: https://creativecommons.org/licenses/by-nc-nd/4.0/.

\section{References}

1. Mieli-Vergani G, Vergani D, Czaja AJ, et al. Autoimmune hepatitis. Nat Rev Dis Primers 2018;4:18017.

2. Czaja AJ. Diagnosis and Management of Autoimmune Hepatitis: Current Status and Future Directions. Gut Liver 2016;10:177-203.

3. Chinese Society of Hepatology, Chinese Society of Gastroenterology \& Chinese Society of Infectious Diseases. Chinese consensus on the diagnosis and management of autoimmune hepatitis (2015). J Digest Dis 2017;18:247-64.

4. Alvarez F, Berg PA, Bianchi FB, et al. International Autoimmune Hepatitis Group Report: review of criteria for diagnosis of autoimmune hepatitis. J Hepatol 1999;31:929-38.

5. Sebode M, Weiler-Normann C, Liwinski T, et al. Autoantibodies in Autoimmune Liver Disease-Clinical and Diagnostic Relevance. Front Immunol 2018;9:609.

6. Mack CL, Adams D, Assis DN, et al. Diagnosis and management of autoimmune hepatitis in adults and children: 2019 practice guidance and guidelines from the American Association for the study of liver diseases. Hepatology 2020;72:671-722.

7. European Association for the Study of the Liver. EASL Clinical Practice Guideline: Autoimmune hepatitis. J Hepatol 2015;63:971-1004.

8. Terziroli Beretta-Piccoli B, Mieli-Vergani G, et al. The clinical usage and definition of autoantibodies in immunemediated liver disease: A comprehensive overview. J Autoimmun 2018;95:144-58.

9. Zachou K, Weiler-Normann C, Muratori L, et al. Permanent immunosuppression in SLA/LP-positive autoimmune hepatitis is required although overall response and survival are similar. Liver Int 2020;40:368-76. 
10. Efe C, Ozaslan E, Wahlin S, et al. Antibodies to soluble liver antigen in patients with various liver diseases: a multicentre study. Liver Int 2013;33:190-6.

11. Zachou K, Gampeta S, Gatselis NK, et al. Anti-SLA/LP alone or in combination with anti-Ro52 and fine specificity of anti-Ro52 antibodies in patients with autoimmune hepatitis. Liver Int 2015;35:660-72.

12. Kirstein MM, Metzler F, Geiger E, et al. Prediction of short- and long-term outcome in patients with autoimmune hepatitis. Hepatology 2015;62:1524-35.

13. Czaja AJ, Donaldson PT, Lohse AW. Antibodies to soluble liver antigen/liver pancreas and HLA risk factors for type 1 autoimmune hepatitis. Am J Gastroenterol 2002;97:413-9.

14. Kanzler S, Weidemann C, Gerken G, et al. Clinical significance of autoantibodies to soluble liver antigen in autoimmune hepatitis. J Hepatol 1999;31:635-40.

15. Czaja AJ, Shums Z, Norman GL. Frequency and significance of antibodies to soluble liver antigen/liver pancreas in variant autoimmune hepatitis. Autoimmunity 2002;35:475-83.

16. Hennes EM, Zeniya M, Czaja AJ, et al. Simplified criteria for the diagnosis of autoimmune hepatitis. Hepatology 2008;48:169-76.

17. Zhao DT, Liao HY, Zhang X, et al. Human leucocyte antigen alleles and haplotypes and their associations with antinuclear antibodies features in Chinese patients with primary biliary cirrhosis. Liver Int 2014;34:220-6.

18. Bowtell DD. Rapid isolation of eukaryotic DNA. Anal Biochem 1987;162:463-5.

19. Wies I, Brunner S, Henninger J, et al. Identification of target antigen for SLA/LP autoantibodies in autoimmune hepatitis. Lancet 2000;355:1510-5.

20. Manns MP, Czaja AJ, Gorham JD, et al. Diagnosis and management of autoimmune hepatitis. Hepatology 2010;51:2193-213.

21. Grønbæk L, Vilstrup H, Jepsen P. Autoimmune hepatitis in Denmark: incidence, prevalence, prognosis, and causes of death. A nationwide registry-based cohort study. J Hepatol 2014;60:612-7.

Cite this article as: Zhang HP, Liu YM, Li Z, Ma YX, Li LJ, Zhao DT, Lou JL, Gao ZH, Yan HP. Clinical characteristics and HLA genotypes in Chinese patients with anti-SLA/LPpositive autoimmune hepatitis. Ann Transl Med 2021;9(2):153. doi: $10.21037 / \mathrm{atm}-20-8036$
22. Strettell MD, Thomson LJ, Donaldson PT, et al. HLA-C genes and susceptibility to type 1 autoimmune hepatitis. Hepatology 1997;26:1023-6.

23. Umemura T, Katsuyama Y, Yoshizawa K, et al. Human leukocyte antigen class II haplotypes affect clinical characteristics and progression of type 1 autoimmune hepatitis in Japan. PLoS One 2014;9:e100565.

24. van Gerven NM, de Boer YS, Zwiers A, et al. HLADRB1*03:01 and HLA-DRB1*04:01modify the presentation and outcome in autoimmune hepatitis type- 1 . Genes Immun 2015;16:247-52.

25. Doherty DG, Donaldson PT, Underhill JA, et al. Allelic sequence variation in the HLA class II genes and proteins in patients with autoimmune hepatitis. Hepatology 1994;19:609-15.

26. Czaja AJ, Strettell MD, Thomson LJ, et al. Associations between alleles of the major histocompatibility complex and type 1 autoimmune hepatitis. Hepatology 1997;25:317-23.

27. de Boer YS, van Gerven NM, Zwiers A, et al. Genomewide association study identifies variants associated with autoimmune hepatitis type 1. Gastroenterology 2014;147:443-52.e5.

28. Qiu DK, Ma X. Relationship between human leukocyte antigen-DRB1 and autoimmune hepatitis type I in Chinese patients. J Gastroenterol Hepatol 2003;18:63-7.

29. Stephens C, Castiella A, Gomez-Moreno EM, et al. Autoantibody presentation in drug-induced liver injury and idiopathic autoimmune hepatitis: the influence of human leucocyte antigen alleles. Pharmacogenet Genomics 2016;26:414-22.

30. Djilali-Saiah I, Fakhfakh A, Louafi H, et al. HLA class II influences humoral autoimmunity in patients with type 2 autoimmune hepatitis. J Hepatol 2006;45:844-50.

31. Oliveira LC, Porta G, Marin ML, et al. Autoimmune hepatitis, HLA and extended haplotypes. Autoimmun Rev 2011;10:189-93.

(English Language Editor: R. Scott) 


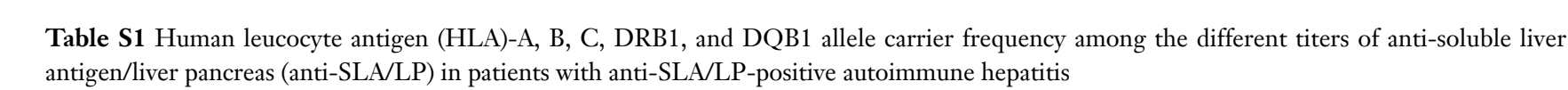

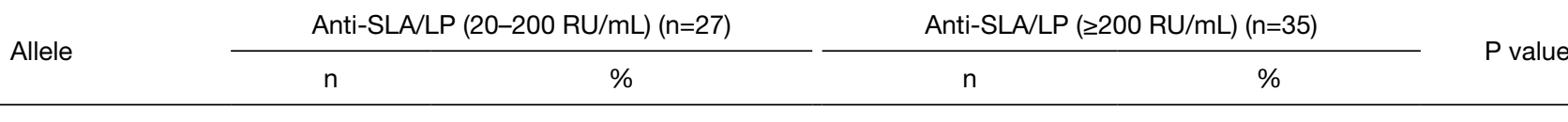
\begin{tabular}{llllll}
\hline$A^{*} 01: 01$ & 0 & 0.0 & 4 & 11.4 & 0.1253 \\
$A^{*} 22: 01$ & 9 & 33.3 & 10 & 28.6 & 0.7837 \\
$A$ & 1 & 37 & 2 & 57 & 1000
\end{tabular} $\begin{array}{llllll}A^{*} 22: 03 & 1 & 3.7 & 2 & 5.7 & 1.0000 \\ A^{*} 22: 05 & 1 & 3.7 & 0 & 0.0 & 0.4355 \\ A^{*} 0200 & 3 & 1.1 & 4 & 1.4\end{array}$

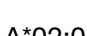
A*02:10 A'03:01 $A^{* 11} 102$ A:24:02 $A=24: 03$

A $25: 01$

A'26:0

A $31: 01$

A'32:01

B.08:01

B"13:01

B"15:01

B"15:02

$B^{*} 15: 18$

B"15:25

B'27:05

B'35:01

B"39:01

C.01:02

c'02:02

c.03:02

c:03:03

C.04:03

407.04

c:08:01

("08:03

C"44:02

C"44:03

DRB1"01:01

DRB'"03:01

DRB 104:03

DRB1" $04: 05$

DRB 104:06

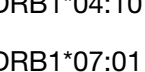

DRB1*08:0

DRB1'08:03

DRB'"09:01

DRB1+11:01

DRB1"12:01

DRB114:02

DRB1"13:03

DRB1"14:01

DRB1"14:04

DRB1"14:05

DRB114:07

DRB1"14:54

DRB1145:02

DRB ${ }^{14} 16: 02$

DQB 1'02:01
DOB 102:02

DQB1*03:0

DQB 1×03:02

DQB 1003:03

B1104:02

QB1105:01

QQB1 $105: 02$

DQB 105:03

DQB $106: 02$

DQB1*06:04

DQB $1 \times 16$ :02

; P<0.05.

0.4355
1.0000

0.2198

1.0000

1.0000

0.4355

0.1856

0.4355
1.0000

1.0000
1.0000

1.0000

$0.0168^{\circ}$

1.0000

1.0000

1.0000
0.1856

0.2504

0.4355

1.0000

0.5003
0.7604

1.0000

0.0003

1.0000

1.0000

0.3088

0.7422

0.4355

0.4355

0.3088

0.5752

1.0000

0.7422

0.4355

0.5003

0.7604

0.3209

0.7351
0.4355

0.5263

0.1856
1.0000

1.0000

0.4182
1.0000

1.0000
0.5752

0.5003

1.0000
0.4355

1.0000

1.0000
0.7192

1.0000

1.0000
1.0000

1.0000
0.5003

0.7604
1.0000

1.0000
1.0000

0.7351

0.4355
0.4550

1.0000
1.0000

1.0000
0.5752

0.6256
10000

1.0000
0.4355

0.4355
0.5003

0.5003
1.0000

0.4355

1.0000
0.4355

0.455
0.0000
0.4851

0.4808

0.6886
1.0000

1.0000

0.7796

0.0000
1.0000

1.0000

1.0000

1.0000
1.0000

0.5752

0.7604

0.2789

0.4355
1.0000 1.0000 


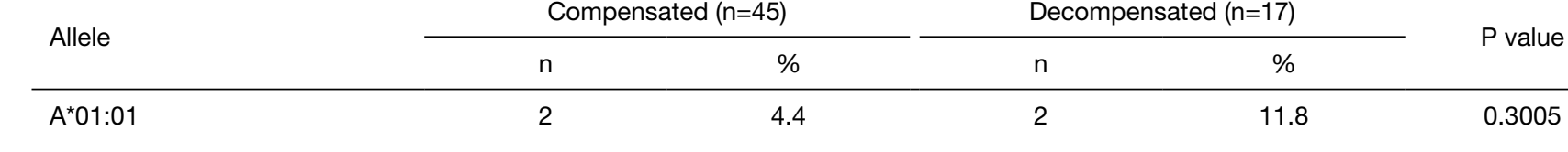

\begin{tabular}{llllll}
\hline$A^{\circ} 01: 01$ & 2 & 4.4 & 2 & 11.8 & 0.3005 \\
$A^{0} 02: 01$ & 17 & 37.8 & 2 & 11.8 & 0.0652 \\
$A^{*} 02: 03$ & 2 & 4.4 & 1 & 5.9 & 1.0000
\end{tabular}

A $02: 05$

$A^{*} 02: 07$

$A^{*} 02: 10$

A*03:01

A"03:02

A $11: 02$

A'24:02

A'24:07

$A^{A} 25: 01$

$A^{2} 26: 01$
$A^{2}=30.01$

$A^{* 31: 01}$

A $32: 01$

$A^{*} 33: 03$
$B^{*} 08: 01$

$B^{*} 13: 01$
$B^{*} 13002$

B"13:02

B"15:01

$\mathrm{B}^{4} 45: 02$
$\mathrm{~B}^{4}+1511$

$B^{*} 15: 11$

B'15:25

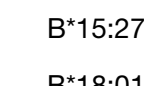

$B^{*} 18: 01$
$B^{*} 27: 05$

B'35:01

B'35:02

B'35:03

$\mathrm{B}^{*} 37: 01$
$\mathrm{~B}^{3} 39: 01$

$\mathrm{B}^{*} 39: 01$
$\mathrm{~B}^{*} 40: 01$

$B^{*} 40: 01$
$B^{4} 40: 02$

$B^{4} 40: 02$
$B^{4} 40: 03$

B'44:03

$\mathrm{B}^{\mathrm{B}} 46: 01$

$B^{8} 48: 01$

$B^{8} 49: 01$
$B^{2}: 1501$

B'51:01
B'51:02

B'52:01

B'54:01

B'58:01

B`67:01
C"01:02

C01:02
$C^{* 0103}$

C.02:02

$\mathrm{C}^{\circ} 03: 02$

c‘03:03

c.03:04

$\begin{array}{ll}c & 04040 \\ C \cdot 04: 03 & \end{array}$

c०04:03

C'07:01

C'07:02

c"07:04

c.08:00
c.08:03

$c \times 08: 03$
$c " 12: 02$

C"12:03

C"14:03

C"15:02

DRB1" $03: 01$

DRB 1"04:01

DRB1"04:02

DRB 1040404

DRB1 104:05

DRB' $104: 10$

DRB' $107: 01$

DRB1"08:03

DRB' $109: 01$

DRB1"11:01

DRB1"12:01

DRB114:13:22

DRB1"13:03

DRB $114: 01$

DRB1"14:03

DRB1"14:04
DRB1"14:05

DRB $1414: 05$

DRB 114:54

DRB14 $15: 01$

DRB 1"145:02
DRB 1416:02

QBB'102:01

DQB 1 $02: 02$

DQB1*03:0

DQB1 103:02

DQB 1104:01

DQB 1×04:02

DQB1‘05:01

DaB1 105:03

DQB1*0:01

DQB $100: 02$

;, P<0.05. 
